\title{
Characteristics of Micromegas detectors at high temperature
}

\section{Longo*}

INFN sezione di Lecce and Università del Salento

E-mail: luigi.longo@cern.ch

\section{J. Samarati}

CERN

E-mail: jerome.samarati@cern.ch

\section{P. lengo}

CERN

E-mail: paolo.iengo@cern.ch

\section{J. Wotschack}

CERN

E-mail: joerg.wotschackecern.ch

The behaviour of two small resistive-strip bulk-Micromegas detectors has been studied up to a temperature of approximately $55^{\circ} \mathrm{C}$. A strong increase of the dark current is observed at temperatures higher than 40 degrees. Keeping the gas at room temperature and varying the detector temperature, the measurements show that the increase of the dark current is primarily related to the detector temperature. The results of a dedicated study of the resistivity of the pillar material as a function of temperature and humidity explain the observed effects. In addition to these studies, the detector response as function of temperature is presented showing that the detector operates correctly up to the highest temperature achieved in this test.

5th International Conference on Micro-Pattern Gas Detectors (MPGD2017)

22-26 May, 2017

Philadelphia, USA

${ }^{*}$ Speaker. 


\section{Introduction}

The temperature studies, addressed in this proceeding, have been performed with two resistivestrip bulk-Micromegas[1], named T1 and T2. Figure 1(a) shows a sketch of the detector layout and Fig. 1(b) shows one of the detectors in the thermal box. Figure 1(c) shows a test board made of a $10 \times 10 \mathrm{~cm}^{2}$ copper area covered with $128 \mu \mathrm{m}$ of coverlay DuPoint ${ }^{\mathrm{TM}}$ Pyralux ${ }^{\circledR}$ PC $1025^{1}$, the same material that is used for the pillars.

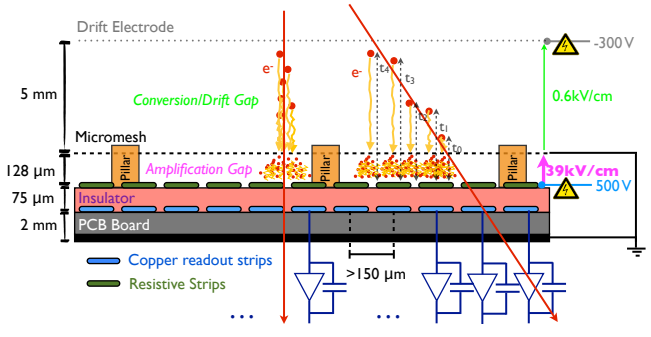

Resistive MM

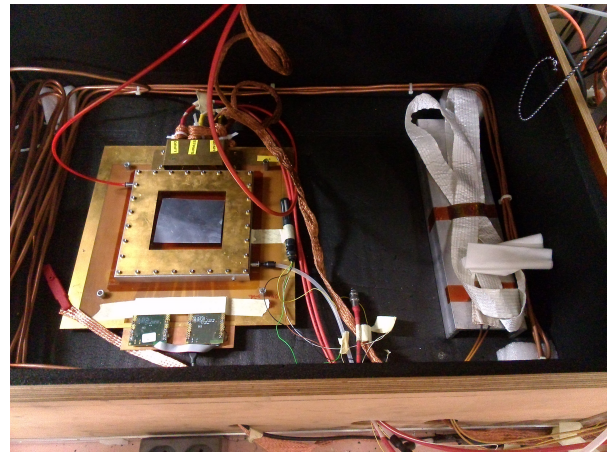

(b)

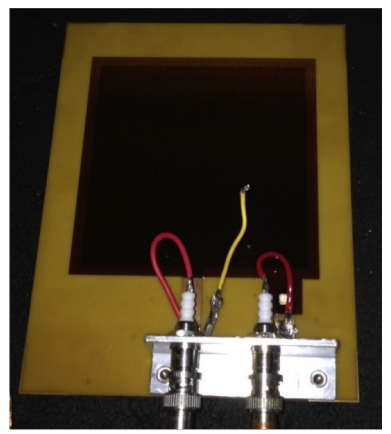

(c)

Figure 1: (a) the sketch of a resistive-strip bulk-micromegas. (b) the wooden insulated box used for the temperature test. Inside the box: on the left the T1 chamber, on the right the thermoelectric strip-device used to increase the temperature. (c) the test board used for the characterization of the pillars material.

Three different types of measurements have been performed. Initially $\mathrm{T} 1$ and $\mathrm{T} 2$ have been warmed up to temperatures higher than $45^{\circ} \mathrm{C}$ to measure the dark current (Sect. 2). Then the Pyralux material has been analyzed, using the test board mentioned before, to understand if changes in humidity and temperature could affect its resistivity (Sect. 3). Finally, using an ${ }^{55} \mathrm{Fe}$ source, the detector response as function of the gas temperature has been investigated (Sect. 4). In the initial and last tests the detectors have been operated with an $\mathrm{Ar}: \mathrm{CO}_{2}$ 93:7 premixed gas at atmospheric pressure.

\footnotetext{
${ }^{1}$ www.dupont.com/content/dam/dupont/products-and-services/electronic-and-electrical-materials/flexible-rigidflex-circuit-materials/documents/PyraluxPC1000coverlay_DataSheet.pdf
} 


\section{Dark current measurements}

The dark current measurements have been performed placing, one at a time, the T-chambers inside the wooden insulated box (Fig. 1(b)), which has been slowly warmed up by a thermoelectric strip-device connected to a potentiometer. In order to avoid gas thermalization, a gas flow of $10 \mathrm{l} / \mathrm{h}$ has been used, like that only the chambers material could be heated. The high voltage (HV) has been applied only to the resistive strips and has been set to $+520 \mathrm{~V}$. The micro-mesh instead has been grounded through a Keithley pico-amperometer, needed to monitor the current coming from the micro-mesh itself. The temperature of the box $\left(\mathrm{T}_{B o x}\right)$ and of the gas $\left(\mathrm{T}_{\text {Gas }}\right)$, at the gas outlet of the chamber, have been monitored together with the atmospheric pressure $(\mathrm{P})$.

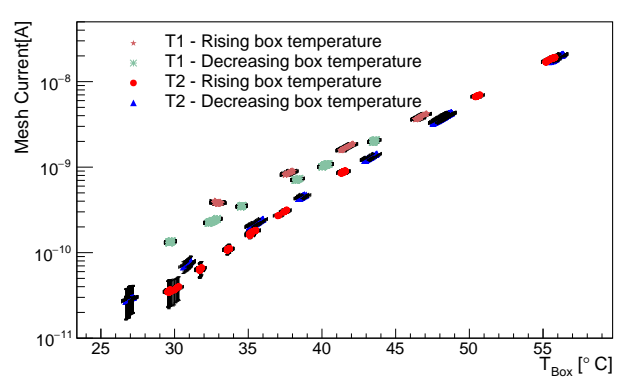

(a)

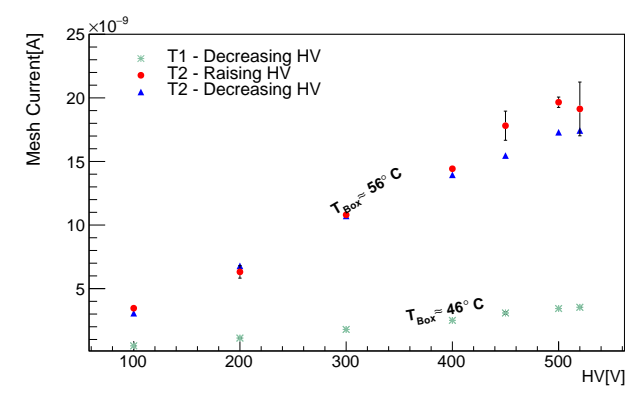

(b)

Figure 2: (a) the dark current observed for $\mathrm{T} 1$ and $\mathrm{T} 2$ while the box temperature is increased or decreased: for both chambers the linear trend on the log scale indicates an exponential growth of the current; no hysteresis effect seems to be present. (b) Mesh current as function of the HV at $46^{\circ} \mathrm{C}$ and $56^{\circ} \mathrm{C}$.

As shown in Fig. 2(a), for both chambers a dark current, growing exponentially as a function of $\mathrm{T}_{\text {Box }}$, has been observed. No hysteresis effect has been noticed, both $\mathrm{T} 1$ and $\mathrm{T} 2$ show approximately the same current behavior increasing and, successively, decreasing the box temperature. Figure 2(b) demonstrates that the material, causing the dark current, must be ohmic. The HV scans, at $\mathrm{T}_{\text {Box }}$ of $46^{\circ} \mathrm{C}$ and of $55^{\circ} \mathrm{C}$, show a linear correlation between the $\mathrm{HV}$, applied to the resistive strips, and the measured current. It is therefore clear that a possible responsible of this behaviour is the Pyralux material used for the pillar construction.

\section{Temperature and humidity tests of the pillar material}

Using the test board shown in Fig. 1(c), the resistivity $(\rho)$ of the Pyralux material has been measured in different conditions of temperature and humidity. In Fig. 3 and in Tab. 1 it is shown that $\rho$ decreases with increasing humidity or temperature. In particular the temperature dependence shows an exponential behaviour that can explain the exponential growth of the dark current. 


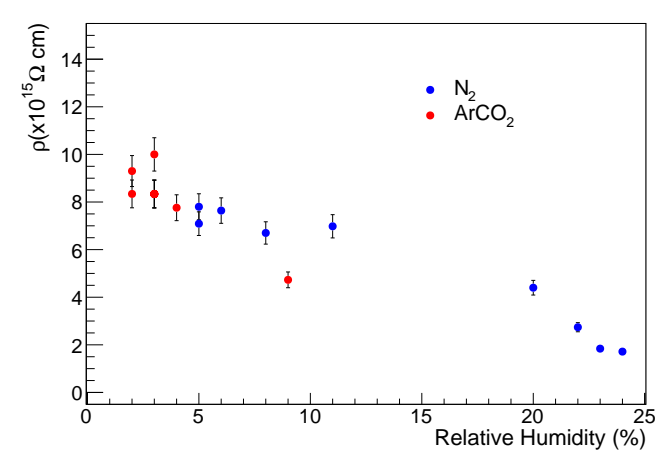

Figure 3: Test board resistivity as a function of the ambient relative humidity: drying the test board with $\mathrm{ArCO}_{2}$ or $\mathrm{N}_{2}$ has given the possibility to gain one order of magnitude with respect to the initial value.
Table 1: Test board resistivity as function of the temperature: the resistivity decreases in agreement with what has been observed in $\mathrm{T}$ Chambers.

\begin{tabular}{cc}
\hline$T_{\text {Box }}\left[{ }^{\circ} \mathrm{C}\right]$ & $\rho(\Omega \mathrm{cm})$ \\
\hline$\approx 20($ Lab temperature $)$ & $\approx 5 \times 10^{14}$ \\
$\approx 29$ & $\approx 1.2 \times 10^{14}$ \\
$\approx 38.3$ & $\approx 3 \times 10^{13}$ \\
$\approx 48.5$ & $\approx 6.8 \times 10^{12}$ \\
\hline
\end{tabular}

Moreover it is interesting to see that the nominal resistivity value, provided by the datasheet $\left(3.4 \times 10^{16} \Omega \mathrm{cm}\right)$, has been roughly reached only in an almost completely dry atmosphere.

\section{Detector performance}

The T1-chamber has been used to check the detector performance at high temperature. As for the dark current measurement, the chamber has been warmed up inside the wooden insulated box but, in this case, applying HV also to the drift electrode $(-300 \mathrm{~V})$ and using a gas flow of $0.3 \mathrm{l} / \mathrm{h}$ to be sure about the gas thermalisation. $\mathrm{P}, \mathrm{T}_{G a s}, \mathrm{~T}_{B o x}$ have been constantly monitored. Photon from an iron-55 source have been used and their energy spectrum has been acquired via a multi-channel analyzer (MCA8000D). For each ${ }^{55} \mathrm{Fe}$ spectrum, a fit has been performed to evaluate the main peak position, $x_{\text {peak }}$, and its FWHM (Fig. 4(a)). Figure 4(b) shows that $x_{\text {peak }}$ increases linearly up to the Pyralux glass-transition temperature, $T_{g} \approx 45^{\circ} \mathrm{C}$, once the effect of the atmospheric pressure has been taken into account. The corresponding effect on the gain can be seen in Fig. 5(a), where an increase of $55 \%$ is observed varying the temperature from $20^{\circ} \mathrm{C}$ to $45^{\circ} \mathrm{C}$. Together with the gain increase also an improvement in the energy resolution of the $8 \%$ up to the glass temperature $\left(T_{g}\right)$ is visible (Fig. 5(b)).

\section{Conclusion}

The temperature behaviour of two small resistive micromegas detectors has been studied. Both detectors give consistent results. The test showed a dark current, growing exponentially with the detector temperature at fixed gas temperature, without any hysteresis effect. This behaviour is explained by the properties of the pillar material; it has been verified that its resistivity decreases strongly with growing temperature and increased humidity. However, the detector performance is not affected, both chambers have been able to operate at temperatures higher than $40^{\circ} \mathrm{C}$, with a peak temperature of the order of $55^{\circ} \mathrm{C}$. A linear growth of the gain has been observed together with an improvement in the energy resolution up to the coverlay glass-transition temperature. 


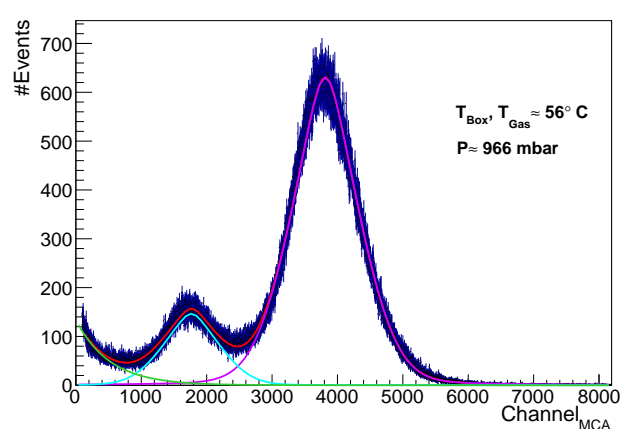

(a)

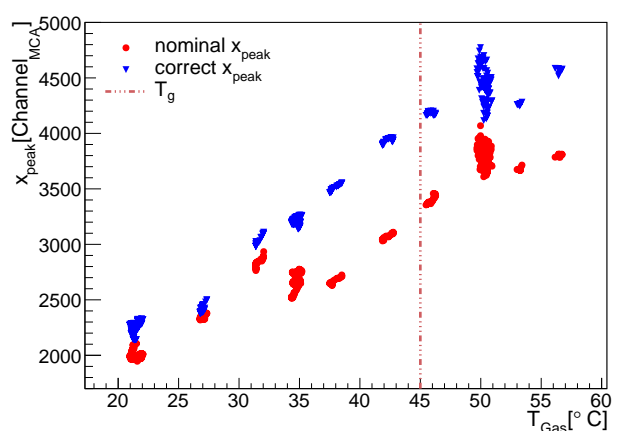

(b)

Figure 4: (a) an iron-55 source spectrum fitted with an exponential function (green curve), to take into account the background component, and two Gaussian plus Cauchy distributions for the escape and main peak description (cyan and violet curves respectively); the final total curve is shown in red. (b) the main peak position as a function of the gas temperature; the red points are obtained directly from the fit, the blue points are the same data after having corrected the gain for the atmospheric pressure change in the Korff approximation, assuming an initial value for the pressure of 950 mbar.

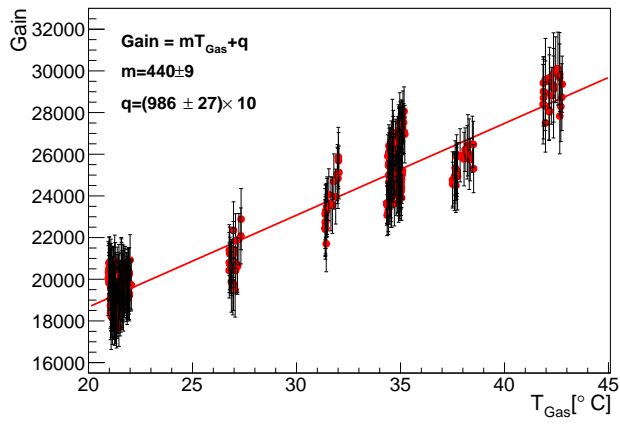

(a)

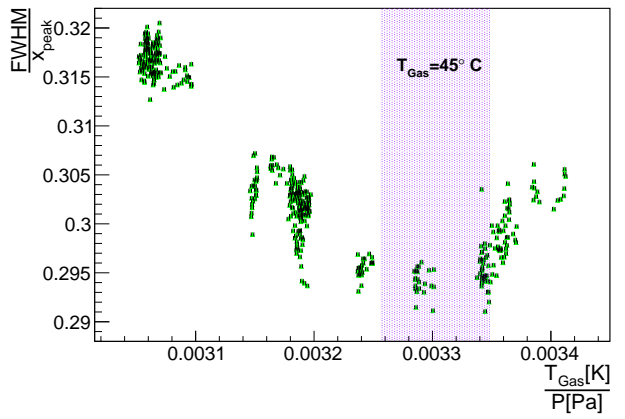

(b)

Figure 5: (a) the gain as a function of the gas temperature. (b) the energy resolution as a function of $T_{G a s} / P$; the shaded area represents the region where $T_{G a s}$ is approximately $45^{\circ} \mathrm{C}$.

\section{Acknowledgments}

The authors would like to thank the CERN RD51 GDD laboratory for their continuous support of our studies.

\section{References}

[1] T. Alexopoulos et al.; A spark-resistant bulk-micromegas chamber for high-rate applications, Nucl. Instrum. Meth. A 640 (2011) 110.

[2] https://twiki.cern.ch/twiki/bin/view/AtlasPublic/NSWPublicResults. 